\title{
High-resolution multistage FWI and image-guided tomography to resolve ultraslow gas anomalies
}

Guy Hilburn, Jian Mao, Jianming Sheng and Jefferson M. Santos

Copyright 2019, SBGf - Sociedade Brasileira de Geofísica

This paper was prepared for presentation during the $16^{\text {th }}$ International Congress of the Brazilian Geophysical Society held in Rio de Janeiro, Brazil, 19-22 August 2019.

Contents of this paper were reviewed by the Technical Committee of the $16^{\text {th }}$ International Congress of the Brazilian Geophysical Society and do not necessarily represent any position of the SBGf, its officers or members. Electronic reproduction of storage of any part of this paper for commercial purposes without the written consent of the Brazilian Geophysical Society is prohibited.

\begin{abstract}
A Celtic Sea survey is processed using a novel workflow combining high-resolution full-waveform inversion (FWI) and tomography, with an emphasis on resolving velocity model anomalies. Gas pockets in shallow sediments cause significant disruption of imaging that continues for kilometers beneath the kinematic anomalies, leading to sagging events and signal attenuation which destroys high frequencies. The model heterogeneities related with these anomalies are resolved through a multistage FWI flow utilizing both dynamic warping and image-guided methods to insert model contrasts, along with highresolution tomography to ensure correct consideration of larger-scale traveltime errors. Final imaging results show dramatically improved event continuity and focusing both in the anomalous areas and in deeper sediments of interest for exploration.
\end{abstract}

\section{Introduction}

Obtaining an accurate velocity model is the key to successful depth processing for most seismic imaging projects. Often there is a high degree of correlation between the geology of a region and its final velocity model; velocity features tend to tie strongly to structure, at least for the imaged large-scale trends. This is not the case in situations where heterogeneities in the subsurface lead to anomalies in seismic models. These are often observed around gas pockets in shallow sediments, which lead to anomalously-low velocities and strong signal attenuation, particularly for high frequencies. When this situation arises, distortions often pervade the image much deeper than the bounds of the kinematic anomalies themselves. Resolving these low velocities and their strongly-contrasted boundaries is vital to improve deeper imaging.

These imaging concerns are of vital importance for a recent offshore Ireland survey which shows strong velocity and attenuation anomaly signatures in the shallow sedimentary layers. While the upper bounds of these regions are imaged, the deeper signal is heavily distorted, and image gathers showed complex and broken events which left little hope for reflection-based tomographic workflows. The successful FWI method presented here utilizes refraction and reflection information to provide high-resolution velocity updates
(Yoon et al., 2014) by gradually increasing the update resolution as details are added to the model. FWI is supplemented with an image-guided tomography (IGT) approach which helps generate contrast as well as constraining the bulk traveltime errors (Hilburn et al., 2014a). The resulting velocity model is geologically-tied through much of the region but has high-contrast features which correspond well with the imaged anomalies. The structure underlying the anomalies shows significant uplift well below their kinematic extents.

The successful hybrid multistage FWI and IGT model building method is discussed, and its application to the presented case study is examined to demonstrate its capabilities.

\section{Method}

FWI is a model building tool for producing high-resolution velocity model updates from comparisons between observed and synthesized seismic data. However, it also suffers from instability and inaccuracy if the proper FWI approach is not applied at the appropriate time in the model building process. Mao et al. (2016) present a multistage $\mathrm{FWI}$ workflow which helps mitigate some of these issues. With this innovative method, early FWI passes use refracted diving wave data, as is common throughout the industry, but the data is dynamically warped (Ma and Hale, 2013) to simplify event-matching. This process helps avoid the cycle-skipping errors prevalent when the current model is far from the true model. Once the shallow model has converged at later stages of model building, the FWI technique shifts its focus by gradually including additional reflection data. To ensure correct positioning of these higher-resolution updates, an image-guided smoothing process is used to encourage the updates to reflect underlying geology. Deeper updates necessitate the use of reflected events, as diving waves cannot penetrate far into the subsurface. However, the conventional reflection FWI approach minimizes the L2 data residual norm, which often poses challenges for noisy, real world data. To stabilize the update in this case, a phase-only reflection FWI method is chosen. Whereas the conventional FWI will often suffer from overcorrection due to its dependence on amplitude information, the phase-only method utilizes a pseudodensity modeling step to better isolate impedance contrasts into velocity or density updates.

This multistage FWI approach avoids many of the inherent difficulties in applying FWI to real data and can produce realistic model contrasts at both imaged and kinematic interfaces. This will undoubtedly help resolve differential moveout between image points. However, a model built purely with FWI is likely to show noticeable residual moveout (RMO) in common image gathers 
(CIGs) due to remaining traveltime errors, leading to poorly focused events at incorrect depths, suggesting that a complementary model building step is required.

Traveltime tomography is a ray-based model building method which uses picked RMO on CIGs to improve event focusing. For this project, the IGT scheme utilizing offset-dependent nonparameterized moveout picking and image-guided update conditioning was chosen (Hilburn et al., 2014b). IGT uses the most recent migrated image to apply appropriate structure-oriented smoothing to the model updates, including automatic edge preservation. During low-resolution, early model building stages, IGT can generate background updates which loosely respect regional geological trends, but with simple parameter changes IGT can also be used to derive high-contrast updates around anomalous regions during later iterations. Due to its separate constraints and ability to create appropriate updates through the entire model building process, IGT is an idea counterpart to the multistage FWI approach.

The hybrid FWI and tomographic model building method works best when each approach can focus on its strengths. The five-iteration workflow for this project proceeds as follows:

1. Long-wavelength IGT generates large-scale velocity features to correct bulk traveltime errors and stabilize later FWI passes.

2. Diving wave dynamic warping FWI creates a higher-resolution update to begin developing stronger model contrasts, while avoiding cycleskipping.

3. IGT is now used to further refine traveltime errors. Stronger edge preservation helps to conserve and strengthen the model contrasts at anomalous regions and layer boundaries.

4. Phase-only image-guided reflection FWI adds high-resolution details to the anomalies and helps insert lateral heterogeneities in the model.

5. A final pass of high-resolution IGT fixes residual traveltime errors and further isolates the imaged anomalous regions.

Alternating passes of FWI and IGT may continue until the desired resolution or convergence is attained, but the final model building iteration should always be a tomographic update to resolve residual traveltime errors.

\section{Examples}

The preceding workflow is demonstrated on the approximately $6,500 \mathrm{~km}^{2} 2017$ narrow azimuth 3D Crean survey, acquired in the Celtic Sea offshore of Ireland. Shallow sediments are layered in an orderly manner, persisting to a prominent chalk layer $3.5 \mathrm{~km}$ deep. Despite being structurally simple the shallow subsurface has several prominent anomalies whose imaging impact is observed up to two kilometers below their upper bounds. Deeper sediments show definite structural sags and low-frequency profiles below these assumed gas pockets, suggesting the initially-smooth velocity needs very strong contrasts at the lateral extents of those anomalies, as can be observed on Figures 1, 2, and 3 . Hart et al. (2015) demonstrated the capability of IGT to build detailed, high-contrast velocity models for similar situations, but early stage reflection data is exceedingly poor around the anomalies. Helpfully, FWI has also shown that it can recover these kinds of model contrasts in areas with poor illumination and low velocities (Yoon et al., 2014).

\section{Results}

Model building proceeds with the five-iteration workflow presented above, alternating passes of IGT and FWI with gradually increasing resolution. FWI adds high-resolution kinematic boundaries where lateral heterogeneities cause sudden changes in the waveform, while IGT produces low-resolution background updates to correct traveltime, then helps enforce imaged model anomalies at later stages.

The benefit of a phase-only reflection FWI method is shown in Figures 4 and 5, which demonstrate the need for an upgraded modeling scheme. In Figure 4, pseudodensity modeling synthetic results resemble the recorded data better than simpler velocity-only modeling, which allows for a better match between the two datasets and more robust model updates. In Figure 5, the new results are compared with the conventional FWI results. The phase-only FWI results in lower magnitude updates which avoid event overcorrection often present in conventional FWI updates, while also being higher in resolution and showing improved adherence to geology.

Uplift in migrated gathers is demonstrated in Figure 6, which depicts CIGs around the strongest gas anomaly. The initial gathers show complicated RMO due to the overly simplified starting model, which leads to difficult and unreliable moveout picking. By proceeding through the workflow presented above, the model converges slowly through low-resolution to high-resolution updates. Each iteration of model building is designed to provide a better starting point for subsequent steps, with the final gathers showing that the complex moveout has been largely corrected and events are better focused across the offset range.

The most significant driver of the overall imaging uplift shown in Figures 1, 2, and 3 is the insertion of an ultraslow velocity in the upper section of the anomaly. This trend is typically $500 \mathrm{~m} / \mathrm{s}$ slower than the surrounding sediments, and it ties strongly to the imaged boundaries of the gas cloud. While the anomalous model updates are constrained to depths shallower than $3 \mathrm{~km}$, imaging uplift is observed to depths greater than $4.5 \mathrm{~km}$, showing the impact that shallow velocity heterogeneities can have on underlying structure. The chalk layer and deeper sediments now appear more geologically plausible, with continuous, focused events, though their low-frequency character remains.

\section{Conclusions}

A successful approach to hybrid high-resolution multistage FWI and tomographic model building allows each method to play to its strengths to overcome the other's weaknesses. FWI can generate strong model contrasts by considering differential shot gather alignment 
across an event, but this often comes with a trade-off for larger-scale traveltime errors. However, FWI can also be beneficial for low-resolution model updates, if reflected signals are significantly more complicated than diving waves. On the other hand, tomography is an excellent tool for correcting traveltime, and therefore depth, errors on CIGs. A jobdeck which alternates between FWI and tomography allows each to highlight its capabilities, while using its complementary method to help mitigate its shortcomings.

The five-iteration model building workflow described here has been applied to a Celtic Sea survey showing strong gas anomalies in shallow sediments and corresponding deeper image distortions. The hybrid model building method begins with unusable CIGs and produces wellfocused events across the offset range. The highcontrast low velocity model anomalies help resolve the non-geological features observed in deeper sediments, removing sag and improving continuity, which leads to simpler structure despite the highly-detailed model updates.

\section{Acknowledgments}

We would like to thank the management of TGS first, for permission to present the survey and method detailed within this abstract. We would also like to acknowledge the contributions of many individuals, particularly Connie VanSchuyver, Yang He, Bin Wang, Jean Ji, Zhiming Li, Matt Hart, Simon Baldock, Nick Woodburn, Sandeep Bhamber, Rachel Ward, Eddie Cho, and Adam Hulks.

\section{References}

Hart, M., Adewumi, O., Lang, C., Hilburn, G. and Rodriguez, G. High resolution velocity model building over the Hoop Fault Complex. 77th Annual International Meeting, SEG, Expanded Abstracts, p. 5189-5193, 2015.

Hilburn, G., He, Y., Sherrill, F., Kim, T. and Yan, Z. Image-guided tomography: structure conforming inversion for complex overburden. First Break, V. 32(6), p. 99-105, 2014a.

Hilburn, G., He, Y., Yan, Z. and Sherrill F. High-resolution tomographic inversion with image-guided preconditioning and offset-dependent picking. 76th Annual International Meeting, SEG, Expanded Abstracts, p. 4768-4772, 2014b.

Ma, Y. and Hale, D. Wave-equation reflection traveltime inversion with dynamic warping and full-waveform inversion. Geophysics, V. 78(6), p. R223-R233, 2013.

Mao, J., Sheng, J., Hart, M. and Kim, T. High-resolution model building with multistage full-waveform inversion for narrow-azimuth acquisition data. The Leading Edge, V. 35(12), p. 1031-1036, 2016.

Yoon, K., Vlad, I., Moghaddam, P., Mao, J. and Sheng, J. An application of FWI to deep water Hernando NAZ streamer data in Gulf of Mexico. 76th Annual International Meeting, SEG, Expanded Abstracts, p. 987-991, 2014.

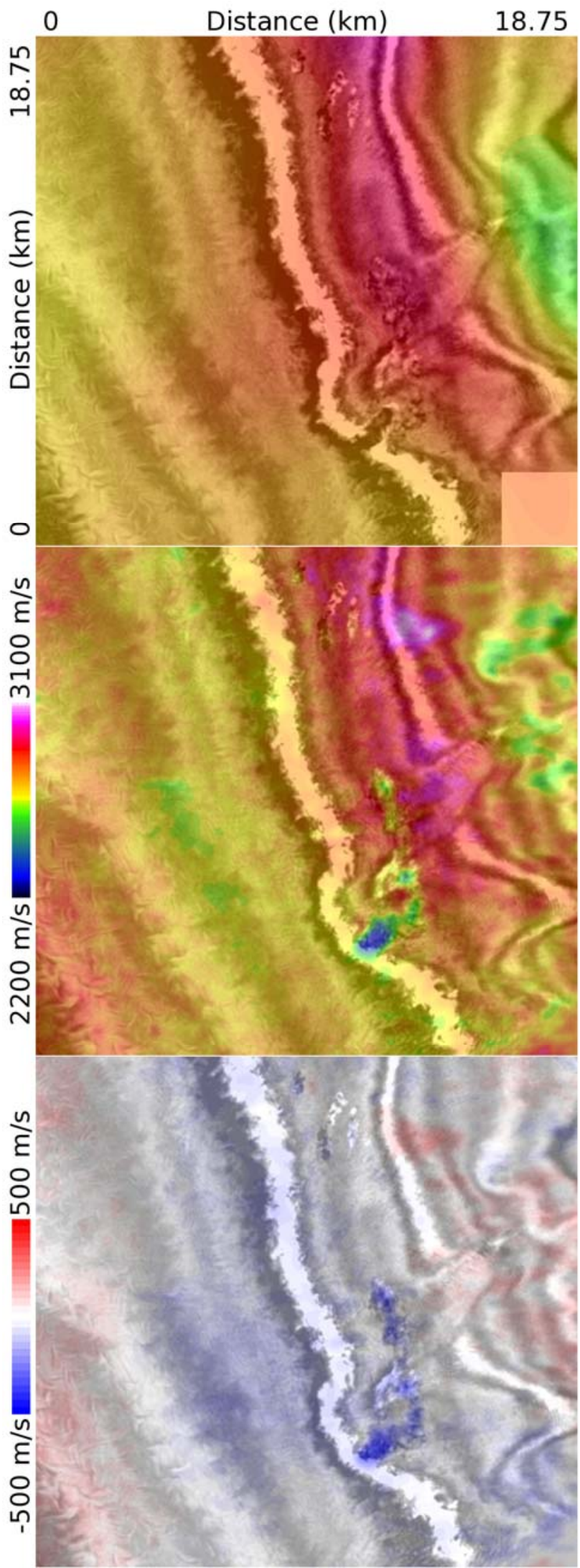

Figure 1 - Depth slices at $2.6 \mathrm{~km}$ of: initial imaging and velocity (top), final imaging and velocity (center), and final imaging and total velocity update (bottom). 


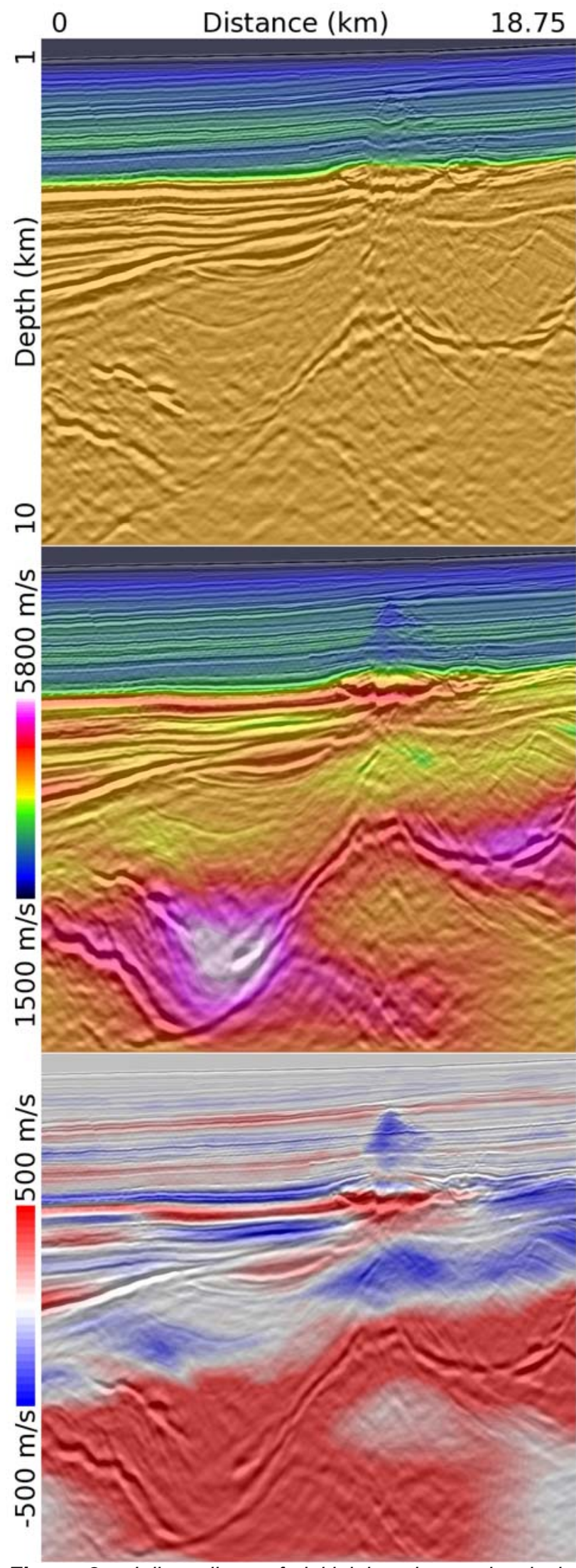

Figure 2 - Inline slices of: initial imaging and velocity (top), final imaging and velocity (center), and final imaging and total velocity update (bottom).

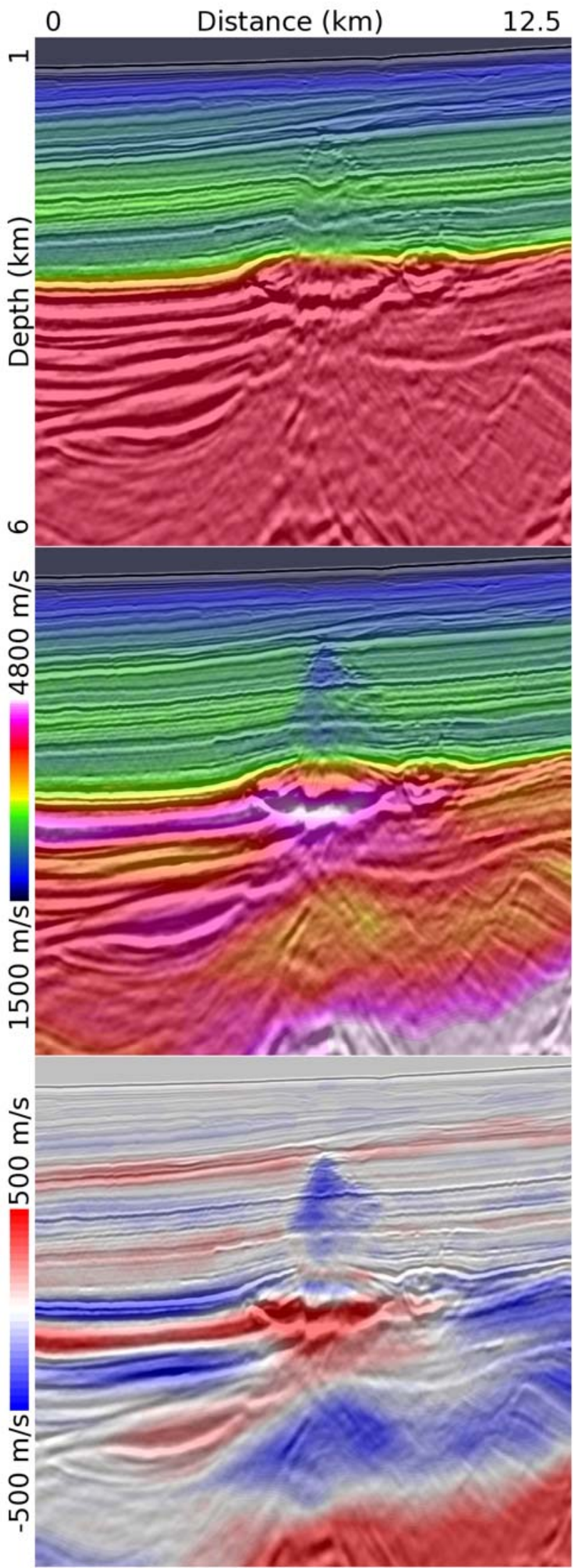

Figure 3 - Zoomed inline slices of: initial imaging and velocity (top), final imaging and velocity (center), and final imaging and total velocity update (bottom). 


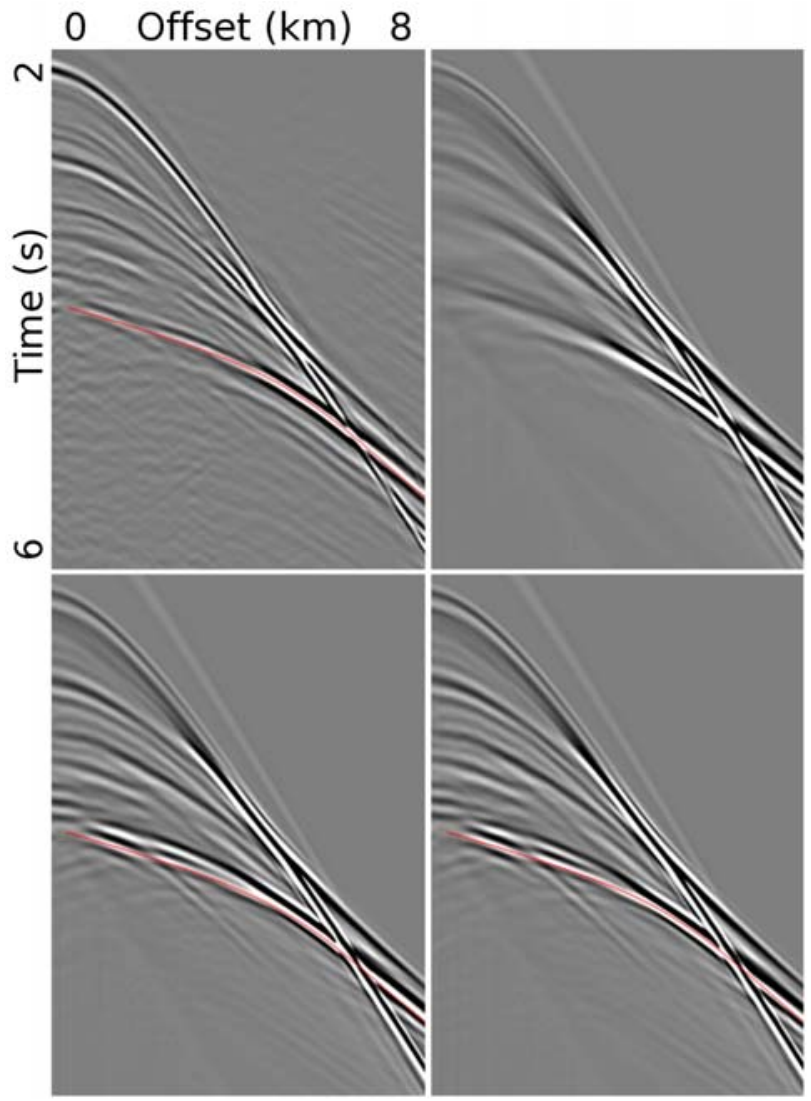

Figure 4 - Input shot gather (top left), initial synthetic shot gather without pseudodensity (top right), initial synthetic shot gather with pseudodensity (bottom left), FWI synthetic shot gather with pseudodensity (bottom right).

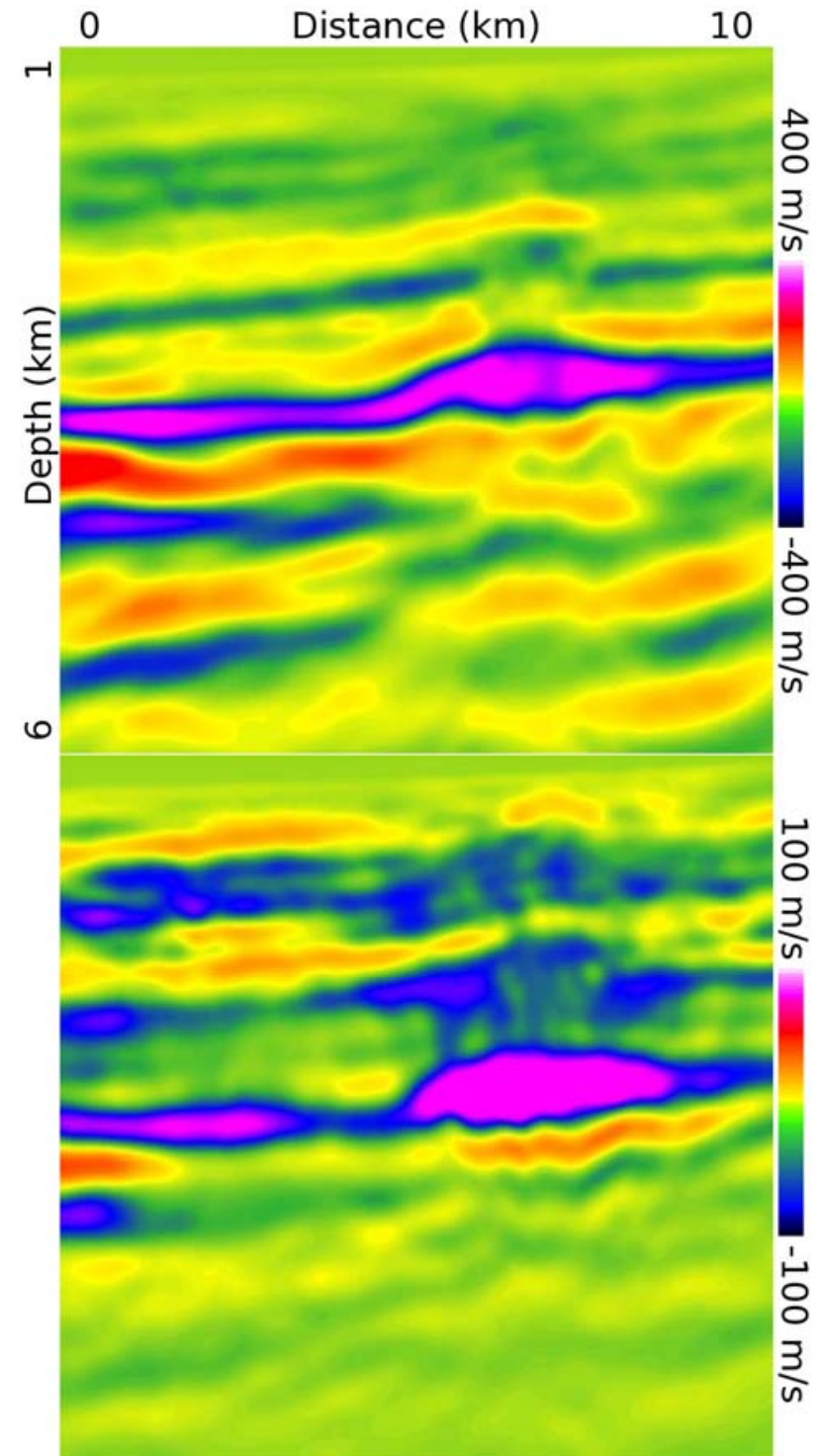

Figure $\mathbf{5}$ - Velocity update from conventional reflection FWI (top) and phase-only reflection FWI (bottom). 


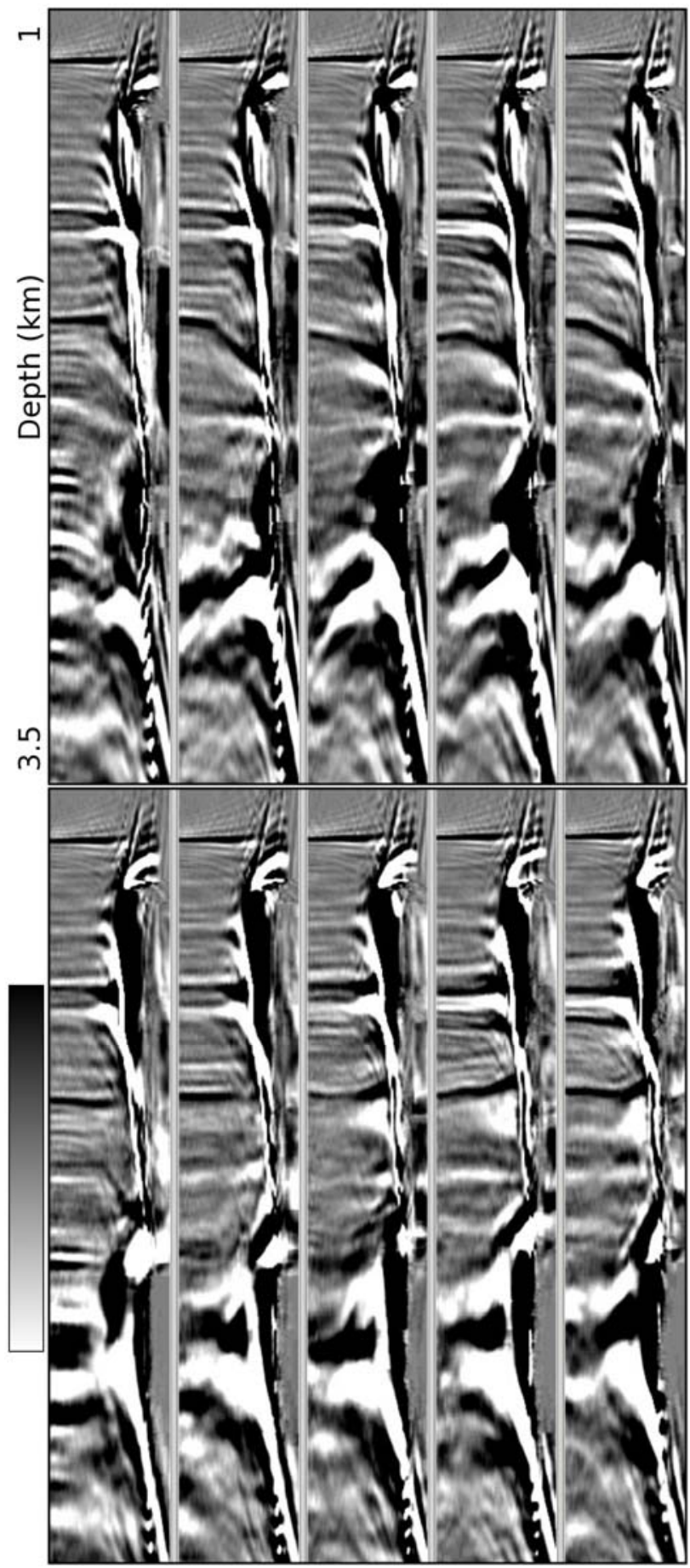

Figure 6 - Common-image gathers around the shallow anomaly before (top) and after (bottom) the hybrid FWItomography approach. 